\title{
Asociación entre estado nutricional y la cantidad de dientes permanentes en niños escolares de Costa Rica
}

\author{
Natalia Gutiérrez-Marín, ${ }^{1}$ (i) Andrea López Soto. ${ }^{2}$ (D)
}

\begin{abstract}
Resumen: El sobrepeso y la obesidad infantil se han convertido en uno de los desafíos de la salud pública a nivel mundial. Estas condiciones pueden afectar varios sistemas, asociándose con un inicio temprano de la pubertad y erupción dental. Objetivo: evaluar si existe una asociación entre el estado nutricional y la cantidad de dientes permanentes erupcionados en una población de niños escolares en Costa Rica. Material y métodos: Estudio transversal con una muestra de 753 niños entre los 6 y 12 años. Se realizó un examen clínico donde se registró la presencia o ausencia de dientes permanentes y se tomaron medidas de peso y talla. El estado antropométrico fue evaluado según los criterios de la Organización Mundial de la Salud. Se efectuó análisis descriptivo para todas las variables, comparación de medias entre los grupos (sobrepeso/obesidad vs peso normal) con la prueba $U$ de Mann-Whitney. Se utilizó un análisis de regresión lineal con la edad y el sexo como covariantes. Resultados: Un 69.20\% niños presentaba un peso normal y 30.80 \% sobrepeso/obesidad. El promedio de dientes erupcionados era mayor en los niños con sobrepreso/obesidad que en aquellos con un peso normal $(p=0.001)$. Al realizar la comparación según el sexo, la diferencia se mantiene (hombres, $p=0.001$; mujeres, $p=0.018$. El análisis de regresión lineal demostró que el estado nutricional $(p=0.001)$ y la edad $(p=0.001)$ están asociadas con el número de dientes permanentes erupcionados. Conclusión: los niños en edad escolar con sobrepeso/obesidad presentan mayor cantidad de dientes erupcionados que sus pares con un peso saludable.
\end{abstract}

Palabras clave: erupción dental, dentición permanente, estado nutricional, sobrepeso, obesidad.

\section{Associação entre o estado nutricional e o número de dentes permanentes em crianças em idade escolar na Costa Rica}

Resumo: O sobrepeso e a obesidade na infância tornaram-se um dos desafios da saúde pública no mundo. Essas condições podem afetar vários sistemas, estando associadas ao início precoce da puberdade e a erupção dentária. Objetivo: Avaliar se há associação entre o estado nutricional e o número de dentes permanentes erupcionados em uma população de escolares da Costa Rica. Materiais e método: Estudo transversal com amostra de 753 crianças entre 6 e 12 anos. Foi realizado un exame clínico onde foi registrada a presença ou ausência dos dentes permanentes e as medidas do peso e altura. $\mathrm{O}$ estado antropométrico foi avaliado de acordo com os critérios da Organização Mundial da Saúde. Fio realizada una análise descritiva para todas as variáveis, comparação das médias entre os grupos (sobrepeso/obesidade vs peso normal) com o teste $U$ de Mann-Whitney. Uma análise de regressão linear foi utilizada como a ideade e o sexo como covariáveis. Resultados: $69,20 \%$ das crianças apresentavam peso normal e 30,80\% sobrepeso/obesidade. O número médio de dentes erupcionados foi maior em crianças com sobrepeso/obesidades $(p=0.001)$. Ao fazer a comparação pelo sexo, a diferença permanece (homens, $p=0.001$; mulheres, $p=0.018$ ). A análise de regressão linear demostrou que o estado nutricional $(p=0.001)$ e a idade $(p=0.001)$ estão associados ao número de dentes permanentes erupcionados. Conclusão: Crianças em idade escolar com sobrepeso/obesas tam mais dentes erupcionados do que seus pares com peso saudável.

Palabra-chave: erupção dentaria, dentição permanente, estado nutricional, sobrepeso, obesidade.

${ }^{1}$ DDS, M. Ed. Especialista en Odontopediatría. Profesora de Odontopediatría, Facultad de Odontología, Universidad de Costa Rica, Costa Rica.

2 DDS, MSc. Profesora, Facultad de Odontología, Universidad Latinoamericana de Ciencia y Tecnología, Costa Rica. 


\section{Association between nutritional status and the number of permanent teeth in a school child in Costa Rica}

Abstract: Childhood overweight and obesity have become one of the public health challenges worldwide. These conditions can affect various systems, they are associated with an early onset of puberty and tooth eruption. Objective: to evaluate whether there is an association between nutritional status and the number of permanent teeth erupted in a population of school children in Costa Rica. Materials and methods: Cross-sectional study with a sample of 753 children between 6 and 12 years old. A clinical examination was performed the presence or absence of permanent teeth weight and height measurements were recorded. The anthropometric status was evaluated according to the criteria of the World Health Organization. Descriptive analysis was carried out for all variables, comparison of means between groups (overweight/obesity versus normal weight) with the Mann-Whitney $U$ test. A linear regression analysis was used with age and sex as covariates. Results: $69,20 \%$ children had a normal weight and $30,80 \%$ were overweight/obese. The average number or erupted teeth as higher in children with overweight/obesity than in those with normal weight $(p=0.001)$. This difference remains in the analysis y sex (men, $p=0.001$; women, $p=0.018$ ). Linear regression analysis demonstrated that nutritional status $(p=0.001)$ and age $(p=0.001)$ are associated with the number of permanent teeth erupted. Conclusion: Overweight/obese school-age children have more erupted teeth than their healthy-weight peers.

Keywords: tooth eruption; dentition permanent; nutritional status; overweight; obesity.

\section{Introducción}

El sobrepeso y la obesidad infantil se han convertido en uno de los desafíos de la salud pública a nivel mundial, según la Organización Mundial de la Salud (OMS) más de 42 millones de niños presentan esta condición y su prevalencia ha incrementado considerablemente en las últimas tres décadas. ${ }^{1}$ El sobrepeso se presenta cuando la ingesta de energía con los alimentos supera las necesidades corporales, resultando en una acumulación de grasa corporal, mientras que la obesidad es una acumulación anormal o excesiva de grasa que puede ser perjudicial para la salud. $^{2}$

La obesidad infantil puede afectarcasi todos los sistemas del cuerpo, por ejemplo, el sistema cardiovascular, musculoesquelético, pulmonar, gastrointestinal y endocrino. ${ }^{3}$
Con respecto a este último, se asocia con un crecimiento acelerado, una maduración esquelética avanzada y un inicio temprano de la pubertad. ${ }^{4}$ En relación con la cavidad oral una gran mayoría de estudios han señalado que los niños con obesidad tienen más dientes erupcionados que sus pares no obesos. ${ }^{5-7}$ Esta posible correlación positiva también se ha documentado en una revisión sistemática publicada en el año $2021 .^{8}$

Dicha correlación positiva se debe a que la erupción puede estar determinada por factores biológicos y ambientales tales como el crecimiento y el desarrollo corporal los cuales, a su vez, están influenciados genéticamente por las hormonas, los hábitos nutricionales, las tendencias seculares y la clase socioeconómica. ${ }^{9}$

Conocer la edad de erupción de la dentición 
permanentes y su posible relación con la contextura del niño es muy importante para los odontopediatras y ortodoncista ya que la aparición de los primeros molares permanentes tiene un impacto en la oclusión, el riesgo de caries, la salud periodontal y por lo tanto el momento de realizar alguna intervención preventiva u ortodóntica. ${ }^{10}$ Se ha sugerido que niños con obesidad pueden requerir una intervención ortodóntica más temprana debido a una erupción dental adelantada. ${ }^{11}$

Ninguno de los estudios que relacionan la obesidad con la erupción dental se han realizado en Centroamérica. Esta región tiene un trasfondo genético, ambiental y cultural diferente; por lo tanto, el objetivo de esta investigación fue evaluar si existe una asociación entre el estado nutricional y la cantidad de dientes permanentes erupcionados en una población de niños escolares en Costa Rica.

\section{Materiales y método}

\section{Participantes}

Este estudio forma parte de una investigación aprobada por la Vicerrectoría de Investigación de la Universidad de Costa Rica (No 440-B7-348). Fue realizado con los niños escolares del Cantón Central de la Provincia de Cartago. Se seleccionó la provincia de Cartago ya que se ubica en el Valle Central donde se concentra gran parte de la población costarricense. Según datos del Ministerio de Educación Pública, el Cantón Central de Cartago reportaba una cantidad de 7788 niños inscritos. El cálculo de la muestra permite estimaciones con un nivel de confianza del 95\% (error tipo I 5\%, tipo II 20\%). Adicionalmente, la muestra contiene una estimación del 25\% de no respuesta.

El Cantón Central de Cartago cuenta con 18 escuelas, las cuales fueron enumeradas en orden alfabético y se seleccionó una de cada dos escuelas para un total de nueve escuelas (ocho públicas y una privada). En cada escuela se escogió al azar un grupo de cada grado, desde primero hasta sexto grado. Establecidas las escuelas participantes, se envió a los encargados de los niños una carta donde se detallaban los pormenores del estudio y se les invitaba a participar, quienes accedían debían firmar un consentimiento informado ( $y$ un asentimiento informado en el caso de los niños de 12 años) y completar un formulario con el sexo y la fecha de nacimiento del niño.

\section{Criterios de selección}

Los criterios de inclusión fueron niños y niñas de 6 a 12 años en dentición mixta con una puntuación $z$ de índice de masa corporal $(\mathrm{IMC}) \geq 3$ y con consentimiento o asentimiento informado firmado según el caso. El rango de edad se eligió así debido a que en esta población la edad promedio de la erupción del primer diente permanente es a los 6,20 años y la segunda molar permanente a los 12,33 años. ${ }^{12}$ Los criterios de exclusión se aplicaron a la hora de hacer el examen clínico y consistieron en niños con malformaciones de esmalte o dentina, con tratamiento ortodóntico, con extracciones dentales previas o sujetos con condiciones que alteran la erupción dental tal como la displasia ectodérmica. No se excluyeron individuos por condición económica, etnia, religión o condición física o mental. 
Variables del estudio

Las variables del estudio fueron edad, sexo, dientes permanentes erupcionados y estado nutricional.

- Determinación de la edad y sexo

Cada encargado del niño llenó un formulario con la fecha de nacimiento y el sexo de cada participante. La edad fue calculada desde la fecha de nacimiento hasta el día de la valoración en meses. Posteriormente se agruparon en las siguientes categorías de edad ${ }^{13}: 6$ años (72 a 83 meses), 7 años (84 a 95 meses), 8 años (96 a 107 meses), 9 años (108-119 meses), 10 años (120131 meses), 11 años (132-143 meses) y 12 años (144-155 meses).

\section{- Determinación de la erupción dental}

El examen dental fue realizado por una odontopediatra previamente calibrada con un patrón oro, se obtuvo un kappa inter-operador de 0,83. La revisión dental se realizó en el aula utilizando una silla dental portátil con luz, un espejo bucal y siguiendo un protocolo de control de infecciones. Una asistente dental asistió a la odontóloga en la toma de los registros. Se contabilizó como diente erupcionado si alguna parte de este había penetrado la mucosa y era visible clínicamente. ${ }^{14} \mathrm{Se}$ incluyeron todos los dientes permanentes excepto los terceros molares.

\section{- Determinación del estado nutricional}

La toma de datos antropométricos la realizó una asistente calibrada. Dentro del aula, en un espacio amplio que permitía los movimientos del medidor y contaba con adecuada iluminación, se determinaron el peso y la talla de los participantes. El peso se tomó según los parámetros establecidos por la OMS: la persona permaneció de pie sin usar zapatos, portando solamente el uniforme. El peso se tomó utilizando una balanza calibrada, hasta los 100 gramos completos más próximos al equilibrio del fiel de la balanza. Para la toma de la talla, el individuo estaba descalzo, de pie sobre una superficie plana. La talla se registró al milímetro completo.

El índice de masa corporal (IMC) y percentil, se obtuvo con el calculador del Centro de Control y Prevención de Enfermedades de EE.UU. ${ }^{15}$ que utiliza la edad, sexo, peso y altura de cada individuo. Esto debido a que en niños de 6-12 años no se puede realizar la clasificación del estado nutricional comparando directamente el IMC. Los valores utilizados de agrupación fueron: peso saludable (puntuación $z$ de IMC entre percentil $\geq 3$ y $\leq 85$ ) y sobrepeso/obesidad (puntuación z de IMC > percentil 85).

\section{Análisis estadístico}

Los datos se registraron en una hoja de cálculo de Excel (Microsoft, Inc., Redmond, WA, EE. UU.), Se ingresaron y se corrigieron las inconsistencias. Se realizó análisis descriptivo de datos para todas las variables, con cálculo de proporciones y medidas de tendencia central y variabilidad.

Se utilizó la prueba de Shapiro-Wilks para verificar la normalidad y la prueba de Levene para evaluar la igualdad de varianzas. Se empleó la prueba U de MannWhitney para la comparación de medias de dientes erupcionados entre individuos de peso saludable y sobrepeso/obesidad; así como la prueba de Chi cuadrado, para 
la comparación de proporciones ( $p<0,05$, IC 95\%). Además, se realizó un análisis de regresión lineal para examinar la relación entre las variables independientes, utilizando la edad y el sexo como covariantes ( $p<0,05$, IC 95\%). Todos los análisis se desarrollaron en SPSS versión 22.0 (SPSS Inc., Chicago, IL, EE. UU.).

\section{Resultados}

En el estudio se incluyeron un total de 753 participantes que cumplieron con los criterios de selección propuestos. EI promedio de edad fue de 8,93 años (rango: 6 a 12 años), el peso $33,36 \mathrm{~kg}$ y la talla 1,32 metros. La mayoría de los pacientes presentaban un peso normal $(69,20 \%)$, un $51,50 \%$ eran mujeres $(n=388)$ y el $48,50 \%$ hombres $(n=365)$. No se presentó diferencia estadísticamente significativa en la distribución del estado nutricional con respecto al peso $(p=0,301)$ y a la distribución según los grupos de edad $(p=0,173)$ (Tabla 1$)$.

El promedio de dientes erupcionados fue mayor en los niños con sobrepreso/ obesidad que en aquellos con un peso normal, esta diferencia fue estadísticamente significativa $(p=0,001)$. De igual forma al realizar el análisis según el sexo, la diferencia se mantiene (hombres, $p=0,001$; mujeres, $p=0,018$ ) (Tabla 2) (Figura 1).

Los resultados del modelo de regresión lineal indican que la edad $(p=0.001)$ y el grado nutricional $(p=0.001)$ afectan de forma positiva el número de dientes erupcionados (Tabla 3).

Tabla 1. Características de la muestra según el estado nutricional

\begin{tabular}{|c|c|c|c|c|}
\hline \multirow[b]{2}{*}{ Características } & \multirow{2}{*}{$\begin{array}{l}\text { Total de individuos } \\
\qquad(n=753)\end{array}$} & \multicolumn{2}{|c|}{ Grupos } & \multirow[b]{2}{*}{$\mathrm{p}$} \\
\hline & & $\begin{array}{c}\text { Peso normal } \\
(n=521 / 69,2 \%)\end{array}$ & $\begin{array}{c}\text { Sobrepeso/obesidad } \\
\quad(n=232 / 30,8 \%)\end{array}$ & \\
\hline Sexo & & & & 0,301 \\
\hline Masculino & 365 (100\%) & $246(67,40 \%)$ & $119(32,60 \%)$ & \\
\hline Femenino & $388(100 \%)$ & $275(70,90 \%)$ & $113(29,10 \%)$ & \\
\hline Edad (promedio, años) & 8,93(1,91 DS) & $8,86(1,92 \mathrm{DS})$ & $9,07(1,89 \mathrm{DS})$ & 0,174 \\
\hline Grupos de edad (años, meses) & & & & 0,173 \\
\hline $6(72$ a 83$)$ & $105(100,0 \%)$ & $76(72,40 \%)$ & $29(27,60 \%)$ & \\
\hline 7 (84 a 95) & $99(100,0 \%)$ & $71(71,70 \%)$ & $28(28,30 \%)$ & \\
\hline 8 (96 a 107) & $119(100,0 \%)$ & $86(72,30 \%)$ & $33(27,70 \%)$ & \\
\hline $9(108-119)$ & $126(100,0 \%)$ & $87(69,00 \%)$ & $39(310,0 \%)$ & \\
\hline $10(120-131)$ & $112(100,0 \%)$ & $76(67,90 \%)$ & $36(32,10 \%)$ & \\
\hline $11(132-143)$ & $108(100,0 \%)$ & $64(59,30 \%)$ & $44(40,70 \%)$ & \\
\hline $12(144-155)$ & $84(100,0 \%)$ & $61(72,60 \%)$ & $23(27,40 \%)$ & \\
\hline Peso $^{\mathrm{b}}$ (promedio, kg) & $33,36(11,12 \mathrm{DS})$ & 29,4(7,9 DS) & 42,4(11,9 DS) & $0,001^{*}$ \\
\hline Talla (promedio, m) & $1,32(0,13 \mathrm{DS})$ & $1,31(0,13 \mathrm{DS}$ & $1,35(0,13 \mathrm{DS})$ & $0,001^{*}$ \\
\hline
\end{tabular}


Tabla 2. Comparación de promedio de dientes permanentes erupcionados de acuerdo con el estado nutricional.

\begin{tabular}{|c|c|c|c|c|c|c|c|c|c|}
\hline & \multicolumn{3}{|c|}{ Total } & \multicolumn{3}{|c|}{ Masculino } & \multicolumn{3}{|c|}{ Femenino } \\
\hline & $\begin{array}{l}\text { Media } \\
\text { (DS) }\end{array}$ & IC (95\%) & $\mathrm{p}^{*}$ & $\begin{array}{c}\text { Media } \\
\text { (DS) }\end{array}$ & IC (95\%) & $\mathrm{p}^{*}$ & $\begin{array}{c}\text { Media } \\
\text { (DS) }\end{array}$ & IC (95\%) & $\mathrm{p}^{*}$ \\
\hline \multicolumn{10}{|l|}{ 6-12 años } \\
\hline Peso normal & $\begin{array}{l}13.52 \\
(7.41)\end{array}$ & $12,88-14,16$ & 0,001 & $\begin{array}{l}13,04 \\
(6,93)\end{array}$ & $12,17-13,92$ & 0,001 & $\begin{array}{l}13,95 \\
(7,81)\end{array}$ & $13,02-14,88$ & 0,018 \\
\hline SP/obesidad & $\begin{array}{l}16,29 \\
(7,70)\end{array}$ & $15,30-17,29$ & & $\begin{array}{l}16,73 \\
(7,80)\end{array}$ & $15,31-18,15$ & & $\begin{array}{l}15,84 \\
(7,60)\end{array}$ & $14,42-17,25$ & \\
\hline
\end{tabular}

SP: sobrepeso, +Prueba U de Mann-Whitney, ${ }^{*}$ diferencia estadísticamente significativa p <0,05; DS (desviación estándar)

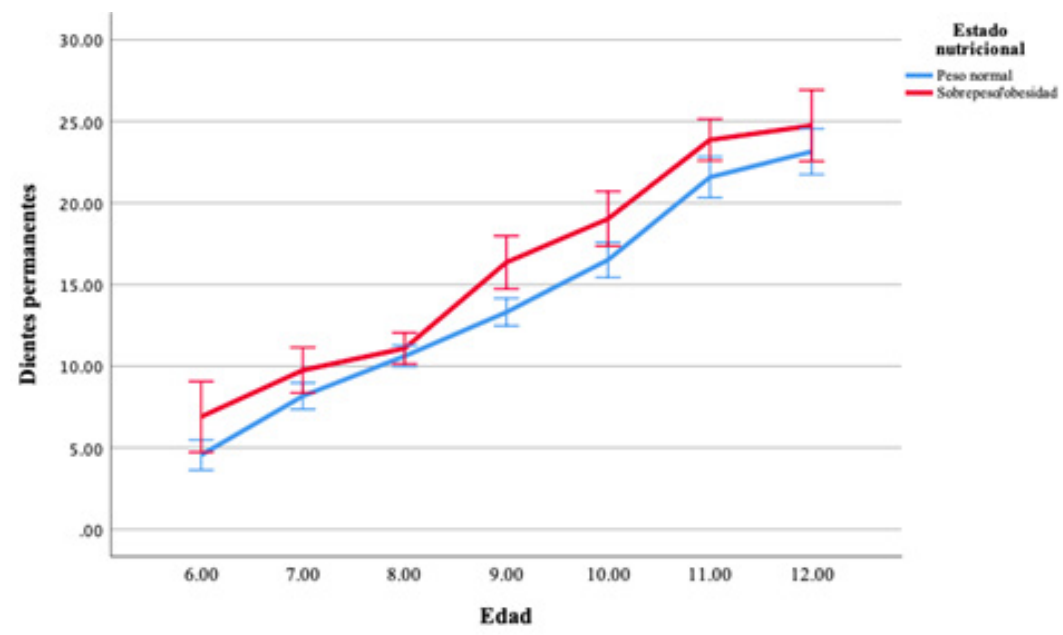

Figura 1. Número (media e intervalo de confianza del 95\%) de dientes permanentes entre niños con sobrepeso/obesidad versus sanos.

Tabla 3. Modelo de regresión lineal de factores que influyen en el número de dientes permanentes erupcionados.

\begin{tabular}{llccc}
\hline \multirow{2}{*}{ Variables } & \multicolumn{3}{c}{ Modelo $\left(\mathrm{R}^{2}=0,69\right)$} \\
\cline { 2 - 5 } Estado nutricional & Beta & $\mathrm{SE}$ & $\mathrm{IC}(95 \%)$ & $\mathrm{p}$ \\
\hline Edad (meses) & 0,8123 & 0,332 & $1,376-2,681$ & $0,001^{*}$ \\
\hline Sexo & $-0,013$ & 0,007 & $0,259-0,286$ & $0,001^{*}$ \\
\hline
\end{tabular}

*diferencia estadísticamente significativa $p<0,05$ 


\section{Discusión}

La obesidad y el sobrepeso son un problema de salud en aumento a nivel mundial, tal como lo demuestra un informe en el año 2021 de la Organización Mundial de la Salud, donde señala que la prevalencia de estas condiciones en niños y adolescentes ha aumentado significativamente, pasando de un $4 \%$ en el año 1975 a un $18 \%$ en el año 2016. ${ }^{16}$ Costa Rica no es la excepción, datos suministrados por el Primer Censo Nacional sobre Peso/Talla en Costa Rica, realizado en el año 2016, indican que un $34 \%$ de los niños tenían sobrepeso/ obesidad. ${ }^{17}$ Siendo esto coincidente con la muestra de esta investigación (30,8\%).

En esta investigación, se determinó que el sobrepeso y la obesidad tienen una correlación positiva respecto al número de dientes permanentes erupcionados. Los dientes permanentes son marcadores biológicos de madurez y su erupción es una señal relevante en el desarrollo de un niño. Diversos factores biológicos y ambientales pueden influir en la edad de erupción de los dientes permanentes, ${ }^{18}$ tal como se estaría mostrando con relación al peso con este trabajo.

Diversos estudios realizados con poblaciones de Brasil,, ${ }^{19}$ África del Sur, ${ }^{20}$ Estados Unidos ${ }^{21}$ y Hong Kong ${ }^{22}$ con edades similares al nuestro concuerdan con que los niños con sobrepeso/obesidad presentan una erupción anticipada de sus dientes permanentes respecto a sus pares con peso saludable. Sin embargo, otros estudios no arrojan resultados concluyentes: un estudio realizado con niños entre los 6 y 8 años no indica una relación positiva, tal situación podría deberse a que la muestra no incluyó niños que ya tuvieran erupcionados premolares y segundos molares permanentes. ${ }^{23}$ Adicionalmente, otras dos investigaciones realizadas no llegan a establecer correlaciones positivas las cuales los mismos autores señalan que pueden deberse a que las muestras fueron pequeñas (300 niños o menos). ${ }^{24,25}$

La erupción temprana de dientes permanentes en niños con sobrepeso/ obesidad puede deberse a que la expansión del tejido adiposo provoca cambios hormonales y metabólicos tales como un aumento de la secreción del factor de crecimiento IGF-1, procesos inflamatorios y metabolismos relacionados con los minerales, los cuales tienen a influir en la erupción dental acelerada. ${ }^{19,26}$ Conocer que los niños con sobrepeso/obesidad pueden tener una mayor cantidad de dientes permanentes es de utilidad ya que lo anterior se ha relacionado con mayor riesgo de caries y una intervención ortodóntica a edades más tempranas. ${ }^{11,27}$ Un meta análisis indica que los niños con esta condición tiene mayor prevalencia de caries en su dentición permanente, siendo más frecuente en los grupos de niños de mayor edad, situación que se ha asociado a una mayor ingesta de azúcares y carbohidratos refinados. ${ }^{28}$ Respecto a las intervenciones ortodónticas, algunos investigadores hacen referencia a que los diferentes tipos de maloclusiones requieren distintos momentos de intervención los cuales están marcados por diversos indicadores de crecimiento y características particulares de cada individuo. ${ }^{29}$

Dentro de las limitaciones de este estudio se encuentra que la muestra a pesar de no ser de conveniencia representa únicamente 
una región de Costa Rica, por lo tanto, no se podría extrapolar los resultados a nivel nacional, sin embargo, la coincidencia de las proporciones de la distribución del estado nutricional con la encuesta nacional podría indicar que los resultados obtenidos estarían dentro de la realidad del país. Por otra parte, no fue posible el análisis segmentando los años según sexo, debido a que el tamaño de la muestra en cada grupo era pequeño, no obstante, tal como se comprueba en la regresión el sexo no es una variable definitoria que influya en el total de dientes permanentes.

Este estudio se realizó de forma transversal por lo que a futuro se planea realizar investigaciones longitudinales donde se incluyan otras variables como el tipo de dieta, el nivel socioeconómico y la presencia de caries, con el fin de dar seguimiento a la población y establecer si los niños con sobrepeso/obesidad que presentan una erupción acelerada, aumentan su riesgo de presentar lesiones cariosas o reciben su tratamiento ortodóntico a tiempo.

\section{Conclusión}

A nivel nacional y Centroamericano es la primera vez que se establece que los niños en edad escolar con sobrepeso/obesidad presentan mayor cantidad de dientes erupcionados que sus pares con un peso saludable. Dicho resultado es similar a los presentados en otras naciones a nivel mundial.

\section{Consideraciones éticas}

Este estudio fue aprobado por la Vicerrectoría de Investigación de la Universidad de Costa Rica (No 440-B7348). El consentimiento y asentimiento informado fue enviado a los padres de cada niño por medio de la administración de cada escuela. Solo los niños con el consentimiento o sentimiento informado firmado pudieron participar del estudio.

\section{Referencias bibliográficas}

1. Organización Mundial de la Salud. Establecimiento de áreas de acción prioritarias para la prevención de la obesidad infantil [Internet]. OMS. Geneva; 2016. Available from: https://www.who.int/dietphysicalactivity/ childhood/tools/es/

2. Sapunarova P, Nihtyanova T, Petrova S, Kukleva M. Association Between Obesity and Permanent Tooth Eruption. J IMAB - Annu Proceeding (Scientific Pap. 2020;26(1):2871-4.

3. Kumar S, Kelly AS. Review of Childhood Obesity: From Epidemiology, Etiology, and Comorbidities to Clinical Assessment and Treatment. Mayo Clin Proc [Internet]. 2017;92(2):251-65. Available from: http:// dx.doi.org/10.1016/j.mayocp.2016.09.017

4. Kaplowitz PB, Slora EJ, Wasserman RC, Pedlow SE, Herman-Giddens ME. Earlier onset of puberty in girls: relation to increased body mass index and race. Pediatrics. 2001 Aug;108(2):347-53.

5. Martínez Cántaro NY. Relación entre el estado nutricional y la cronología de la erupción dental en estudiantes escolares de Tacna. Rev Odontológica Basadrina. 2020;3(2):11-8.

6. Prijatmoko D, Zakiyah F. Role of body composition on the eruption time of first permanent molars. J Int Dent Med Res. 2019;(4):1563-7.

7. Valenzuela Ramos M, Ojeda Gómez R CF. Erupción dental relacionada con el indicador peso para la edad. Av Odontoestomatol. 2018;34(4):193-8.

8. Mohamedhussein N, Busuttil-Naudi A, Mohammed H, UlHaq A. Association of obesity with the eruption of first and second permanent molars in children: a systematic review. Eur Arch Paediatr Dent [Internet]. 2020;21(1):13-23. Available from: https://doi.org/10.1007/s40368-019-00452-6 
9. Arid J, Vitiello MC, da Silva RAB, da Silva LAB, de Queiroz AM, Küchler EC, et al. Nutritional status is associated with permanent tooth eruption chronology. Brazilian J Oral Sci. 2017;16:1-7.

10. Younus MS, Ahmed K, Kala D. The effect of body mass index on tooth eruption and dental caries. Dent J (Majalah Kedokt Gigi). 2020;53(3):140.

11. Nicholas CL, Kadavy K, Holton NE, Marshall T, Richter A, Southard T. Childhood body mass index is associated with early dental development and eruption in a longitudinal sample from the lowa Facial Growth Study. Am J Orthod Dentofac Orthop [Internet]. 2018;154(1):72-81. Available from: https://doi. org/10.1016/j.ajodo.2017.10.033

12. Gutiérrez-Marín N, López Soto A, Castillo Rivas J. Age and Sequence of Emergence of Permanent Teeth in a Population of Costa Rican Schoolchildren. Odovtos - Int J Dent Sci. 2020;325-32.

13. Pahel BT, Vann WF, Divaris K, Rozier RG. A Contemporary Examination of First and Second Permanent Molar Emergence. J Dent Res [Internet]. 2017;96(10):1115-21. Available from: https://www.unicef.org/ costarica/media/2611/file

14. Chaitanya P, Reddy JS, Suhasini K, Chandrika IH, Praveen D. Time and Eruption Sequence of Permanent Teeth in Hyderabad Children: A Descriptive Cross-sectional Study. Int J Clin Pediatr Dent. 2018;11(4):3307.

15. Centers for Disease and Preventation. BMI Calculator Child and Teen | Healthy Weight [Internet]. 2019. Available from: https://www.cdc.gov/healthyweight/bmi/result.html?\&method=english\&gender=m\&age $\mathrm{y}=19$ \&age_m $=0$ \&hft $=6$ \& hin $=0 \& \mathrm{twp}=154.3$

16. Organización Mundial de la Salud. Obesidad y sobrepeso. Obesidad y sobrepeso. 2020.

17. Ministerio de Educación Publica ETNC. Informe ejecutivo: Censo Escolar PEso/Talla Costa Rica 2016. 2016.

18. Subramaniam P, Pagadala R. Association of eruption timing of first permanent molars and incisors with body mass index of children in Bengaluru City. J Indian Assoc Public Heal Dent. 2020;18(1):70-6.

19. Evangelista S e S, Vasconcelos KRF, Xavier TA, Oliveira S, Dutra ALT, Nelson-Filho P, et al. Timing of Permanent Tooth Emergence is Associated with Overweight/Obesity in Children from the Amazon Region. Braz Dent J. 2018;29(5):465-8.

20. Esan TA, Schepartz LA. Does nutrition have an effect on the timing of tooth formation? Am J Phys Anthropol. 2020;171(3):470-80.

21. Must A, Phillips SM, Tybor DJ, Lividini K, Hayes C. The Association Between Childhood Obesity and Tooth Eruption. Obes J [Internet]. 2012;20(10):2070-4. Available from: http://doi.wiley.com/10.1038/ oby.2012.23

22. Wong HM, Peng SM, Yang Y, King NM, McGrath CPJ. Tooth eruption and obesity in 12-year-old children. J Dent Sci [Internet]. 2017;12(2):126-32. Available from: http://dx.doi.org/10.1016/j.jds.2016.10.004

23. Rachmawati E, Murniati N, Febriani M. Description of Permanent Teeth Eruption and Body Mass Index among Elementary School Children of 6 - 9 Years Old in Bandung City . J Dent Med Sci. 2020;19(7):37-41.

24. Díaz-Orahulio GD, León-Manco RA. Estado nutricional y secuencia de erupción dentaria en niños menores de 12 años de edad - Aldea Infantil SOS Pachacámac - Lima, Perú. Rev Estomatológica Hered. 2014;24(4):213.

25. Hassan S, Shahid H. Assesment of Eruption of Permanent Teeth According To Age And Its Relation With Body Mass Index In Local Population. J Pakistan Dent Assoc. 2018;27(03):127-32.

26. Nicholas C, Thalji G, Richter A. Childhood Obesity and Accelerated Timing of Dental Development: A Critical Review. Forensic Anthropol. 2018;1(3):170-9.

27. Šindelářová R, Soukup P, Broukal Z. The relationship of obesity to the timing of permanent tooth emergence in Czech children. Acta Odontol Scand [Internet]. 2018;76(3):220-5. Available from: https://doi.org/10.10 80/00016357.2017.1403649

28. Hayden C, Jo B, Chambers S, Freeman R, Humphris G, Richards D. Obesity and dental caries in children : a systematic review and meta-analysis. Community Dent Oral Epidemiol. 2013;2006(7):289-308.

29. Perinetti G, Franchi L, Contardo L. Determination of timing of functional and interceptive orthodontic treatment: A critical approach to growth indicators. J World Fed Orthod [Internet]. 2017;6(3):93-7. Available from: http://dx.doi.org/10.1016/j.ejwf.2017.08.006

Recibido: $29 / 07 / 2021$

Aceptado: 08/11/2021

Correspondencia: Natalia Gutiérrez-Marín, correo: natalia.gutierrez@ucr.ac.cr 